Transportation Research Record $1-12$

\title{
Planning of Fast-Charging Stations for a Battery Electric Bus System under Energy Consumption Uncertainty
}

(C) National Academy of Sciences: Transportation Research Board 2018 Reprints and permissions: DOI: 10.1 I77/036 I 198118772953 journals.sagepub.com/home/trr (S)AGE

\author{
Zhaocai Liu', Ziqi Song', and Yi He'
}

\begin{abstract}
Battery-powered electric buses are gaining popularity as an energy-efficient and emission-free alternative for bus fleets. However, battery electric buses continue to struggle with concerns related to their limited driving range and time-consuming recharging processes. Fast-charging technology, which utilizes dwelling time at bus stops or terminals to recharge buses in operation employing high power, can raise battery electric buses to the same level of capability as their diesel counterparts in terms of driving range and operating time. To develop an economical and effective battery electric bus system using fastcharging technology, fast-charging stations must be strategically deployed. Moreover, due to the instability of traffic conditions and travel demands, the energy consumption uncertainty of buses should also be considered. This study addresses the planning problem of fast-charging stations that is inherent in a battery electric bus system in light of the energy consumption uncertainty of buses. A robust optimization model that represents a mixed integer linear program is developed with the objective of minimizing the total implementation cost. The model is then demonstrated using a real-world bus system. The performances of deterministic solutions and robust solutions are compared under a worst-case scenario. The results demonstrate that the proposed robust model can provide an optimal plan for a fast-charging battery electric bus system that is robust against the energy consumption uncertainty of buses. The trade-off between system cost and system robustness is also addressed.
\end{abstract}

The electrification of bus fleets has been widely considered as an effective method of abating local emissions, improving fuel economy, and reducing oil dependence in conventional diesel-powered bus systems (1-3). Battery electric buses (BEBs) are among the most popular electric buses today. Compared to trolleybuses, which are attached to continuous overhead wires along the entire bus line, BEBs are more flexible in their operation. However, due to the limitations of battery technology, the energy density of batteries for BEBs is rather low when compared to diesel. BEBs therefore suffer from the disadvantages of cumbersome and costly on-board batteries and limited driving ranges. Moreover, the refueling of BEBs using either standard or slow-charging methods takes much longer when compared to diesel buses. The emerging technology of fast-charging BEBs provides promising potential in helping to offset these drawbacks. This technique follows the opportunity charging concept, which utilizes dwelling times at bus stops or terminals to recharge buses in operation using high charging power (3). By fast-charging en route, BEBs are as capable as their diesel counterparts in terms of range and operating time. Promising results have been reported by many fastcharging BEB demonstration projects (4-6).

A fast-charging BEB system requires comprehensive planning and design to ensure effective and economical implementation. Two key design factors of the system are the deployment of fast-charging stations and onboard battery size. The planning problem is twofold. First, the combination of deployed fast-charging facilities and the design of battery sizes should ensure the normal operation of the bus system. Second, one must consider the trade-off between on-board battery sizes and the number of fast-charging facilities.

Although several studies have investigated the planning problem of a fast-charging electric bus system (e.g., $1,3,7)$, none have considered the energy consumption uncertainty of buses. For a fast-charging electric bus

\footnotetext{
'Department of Civil and Environmental Engineering, Utah State University, Logan, UT
}

Corresponding Author:

Address correspondence to Ziqi Song: ziqi.song@usu.edu 
system, the energy consumption of BEBs is a key parameter in determining the locations of charging stations and the battery sizes of BEBs. In the above studies, energy consumption is assumed to be predefined and is estimated either based on collected sample data or through simulation. In reality, however, the energy consumption of BEBs may be uncertain. For instance, the energy consumption of a BEB between two stops will change in conjunction with traffic conditions and travel demands. Ignoring the uncertainty of energy consumption of BEBs could lead to a suboptimal or even an infeasible plan for a fast-charging electric bus system. Therefore, this study explicitly addresses energy consumption uncertainty in the planning of a fast-charging electric bus system.

To model the energy consumption uncertainty of BEBs, this study applies the technique of set-based robust optimization (RO). This technique has been developed and improved by many researchers (e.g., 8-10). It allows the parameters with uncertainty to vary within a given set and requires solutions to be feasible for any realizations of the parameters in the set. Compared with other modeling methods regarding data uncertainty, such as stochastic programming and scenario-based RO, the set-based $\mathrm{RO}$ has the virtues of not requiring probability distribution of uncertain data and providing computationally tractable formulations. The set-based RO approach has been applied in many study areas, including humanitarian relief supply chain problems with demand uncertainty (11), multi-period fleet allocation problems in a bike-sharing system with demand uncertainty (12), robust dynamic network design problems with demand uncertainty (13), and robust planning of dynamic wireless charging infrastructure for BEBs (14).

In this study, we first provide a deterministic optimization model for the planning problem of a fast-charging electric bus system. We then develop its robust counterpart model to address the energy consumption uncertainty of BEBs. The approach developed by Ben-Tal et al. (10) is adopted to derive the robust counterpart for a given uncertainty set.

\section{Deterministic Optimization Model}

In this section, we introduce the deterministic optimization model for the planning of a fast-charging electric bus system. To develop a feasible and cost-effective system, the battery sizes of BEBs and the deployment of fast-charging stations need to be simultaneously determined. Because there are multiple types of fast-charging stations with different power levels and costs, we also need to determine the most suitable type for each charging station. The combination of designed battery sizes and deployed charging stations should first meet the energy requirements for normal operation of the bus system. Under this premise, we then seek the optimal combination with minimum total cost. Moreover, our model is based on a fast-charging electric bus system with the following assumptions:

1. Each bus line in the bus system operates on a fixed route.

2. Each bus line has a base station, where all buses begin and end each of their service loops. Different bus lines may share a base station if their service routes converge at the base station.

3. After finishing a service loop, an electric bus will be fully charged at the base station before it starts another service loop.

Assumption 1. is not restrictive, because we can always treat a bus line with multiple routes as multiple lines that share the same bus fleet and require the bus fleet to be feasible for all lines. Assumption 2. is accordant with the common practice of bus systems. The base station is usually the location where drivers have a rest and buses are checked. Assumption 3. means that a fast-charging station must be installed at the base station, and that an electric bus will utilize its long dwell time between two service loops to get fully charged. Assumption 3. is consistent with the operational rules in the demonstration project of a fast-charging electric bus line, as reported by Eudy et al. (4). We note that some other studies, such as that of Qin et al. (15), allow electric buses to operate more than one service loop before they are fully charged. However, this may result in a queue at the charging station and require additional scheduling efforts to ensure normal operations. Because the primary focus of this study is to develop a modeling framework for the robust optimal planning of a fast-charging electric bus system, we leave the work of capturing more flexible operational rules to future studies.

\section{Notation}

For the convenience of readers, we list all notations of the deterministic model in Table 1.

\section{Model Formulation}

$$
\begin{aligned}
& (\mathrm{S} 1): \min _{\left(\boldsymbol{x}, \boldsymbol{y}, \boldsymbol{e}^{\max }, \boldsymbol{s}, \boldsymbol{e}^{\text {arriving }}, \boldsymbol{e}^{\text {leaving }}\right)} \sum_{k \in K} a^{\text {battery }} \zeta_{k} e_{k}^{\max } \\
& +\sum_{i \in \tilde{N}} \sum_{t \in T} a_{t}^{\text {charger }} x_{i, t}+\sum_{k \in K} \sum_{i \in N \backslash \tilde{N}} \sum_{t \in T} a_{t}^{\text {charger }} y_{k, i, t} \\
& \text { s.t. } \\
& \sum_{t \in T} x_{i, t} \leq 1 \quad \forall i \in \tilde{N} \\
& \sum_{t \in T} y_{k, i, t} \leq 1 \quad \forall k \in K, i \in N_{k} \backslash \tilde{N}
\end{aligned}
$$


Table I. Notation of the Deterministic Model

\begin{tabular}{|c|c|}
\hline & Description \\
\hline \multicolumn{2}{|l|}{ Sets } \\
\hline$N$ & Set of bus stations (including base stations and terminals), indexed by $i$ and $j$ \\
\hline$L$ & Set of directed road segments connecting sequential stations, indexed by $(i, j)$ \\
\hline$T$ & Set of fast-charging station types, indexed by $t$ \\
\hline$K$ & Set of bus lines, indexed by $k$ \\
\hline$\tilde{N}$ & Set of bus stations (excluding base stations and terminals), indexed by $i$ and $j$ \\
\hline$L_{k}$ & Set of all the links that form bus line $k \in K$ \\
\hline$N_{k}$ & Set of all the nodes that form bus line $k \in K$ \\
\hline \multicolumn{2}{|c|}{ Parameters } \\
\hline$o_{k}$ & A node representing the base station of bus line $k \in K$ \\
\hline$a^{\text {battery }}$ & Amortized battery cost per $\mathrm{kWh}$, considering replacement and discount rate \\
\hline$a_{t}^{\text {charger }}$ & Amortized cost of installing a type $t \in T$ fast-charging station \\
\hline$\zeta_{k}$ & Total number of buses on line $k \in K$ \\
\hline$c_{k, i, j}$ & Energy consumption of an electric bus from line $k \in K$ on link $(i, j) \in L_{k}$ \\
\hline$\tau_{k, i}$ & Dwell time of an electric bus from line $k \in K$ at station $i \in N$ \\
\hline $\begin{array}{l}P_{t} \\
\epsilon^{\text {upper }}\end{array}$ & $\begin{array}{l}\text { Power of a type } t \in T \text { fast-charging station } \\
\text { Upper bound of remaining battery power (in percentage of battery capacity) }\end{array}$ \\
\hline$\epsilon^{\text {lower }}$ & Lower bound of remaining battery power (in percentage of battery capacity) \\
\hline \multicolumn{2}{|r|}{ 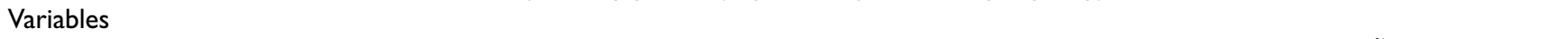 } \\
\hline$x_{i, t}$ & $\begin{array}{l}\text { A binary variable, representing whether to build a type } t \in T \text { fast-charging station at bus station } i \in \tilde{N} \text {. If yes, } x_{i, t}=1 \text {; } \\
\text { otherwise, } x_{i, t}=0\end{array}$ \\
\hline$y_{k, i, t}$ & $\begin{array}{l}\text { A binary variable, representing whether to build a type } t \in T \text { fast-charging station at base station or terminal station } \\
\qquad i \in N \backslash \tilde{N} \text {. If yes, } y_{k, i, t}=1 \text {; otherwise, } y_{k, i, t}=0\end{array}$ \\
\hline \multirow{4}{*}{$\begin{array}{l}\mathrm{e}_{k}^{\max } \\
\mathrm{e}_{k, i}^{\text {arriving }} \\
\mathrm{e}_{k, i}^{\text {leaving }} \\
s_{k, i}\end{array}$} & Battery size for electric buses on line $k \in K$ \\
\hline & Remaining battery power of an electric bus on line $k \in K$ when it arrives at station $i \in N$ \\
\hline & Remaining battery power of an electric bus on line $k \in K$ when it leaves station $i \in N$ \\
\hline & Energy supply at station $i \in N$ for an electric bus from line $k \in K$ \\
\hline
\end{tabular}

$$
\begin{gathered}
x_{i, t} \in\{0,1\} \quad \forall i \in N, t \in T \\
y_{k, i, t} \in\{0,1\} \quad \forall i \in N, t \in T \\
e_{k, i}^{\text {leaving }}=\epsilon^{\text {upper }} e_{k}^{\text {max }} \quad \forall k \in K, i=o_{k} \\
e_{k, j}^{\text {arriving }}=e_{k, i}^{\text {leaving }}-c_{k, i, j} \quad \forall k \in K,(i, j) \in L_{k} \\
e_{k, i}^{\text {leaving }}=e_{k, i}^{\text {arriving }}+s_{k, i} \quad \forall k \in K, i \in N_{k} \\
s_{k, i} \leq \sum_{t \in T} p_{t} x_{i, t} \tau_{k, i} \quad \forall k \in K, i \in N_{k} \cap \tilde{N} \\
s_{k, i} \leq \sum_{t \in T} p_{t} y_{k, i, t} \tau_{k, i} \quad \forall k \in K, i \in N_{k} \backslash \tilde{N} \\
e_{k, i}^{\text {arriving }} \geq \epsilon^{\text {lower }} e_{k}^{\max } \quad \forall k \in K, i \in N_{k} \\
e_{k, i}^{\text {leaving }} \leq \epsilon^{\text {upper }} e_{k}^{\max } \quad \forall k \in K, i \in N_{k} \\
e_{k}^{\max } \geq 0 \quad \forall k \in K
\end{gathered}
$$

The objective function minimizes the total system cost, including the cost of batteries and the cost of installing fast-charging stations. Note that because the dwelling time or the potential charging time at a base station or terminal station is long, to avoid a queue, we assume that different bus lines cannot share charging stations at a shared base station or terminal station. While at a bus station that is neither a base station nor a terminal station, we assume that different bus lines can share one charging station. We note that to realize charging station sharing, a bus system must be systematically and comprehensively scheduled. We leave the work of developing a schedule-based modeling framework to future studies.

Constraint (1) ensures that only one type of fast-charging station can be installed at bus station $i \in \tilde{N}$. Similarly, constraint (2) ensures that for bus line $k \in K$, only one type of charging station can be deployed at the base station or terminal station $i \in N_{k} \backslash \tilde{N}$. Constraints (3) and (4) require variables $x_{i, t}$ and $y_{k, i, t}$ to be binary. Constraint (5) specifies that a BEB is fully charged when it begins its service from the base station. Constraints (6) and (7) describe the change in battery power on a bus due to energy consumption and potential fast-charging. Constraints (8) and (9) ensure that an electric bus can be charged at a bus station only when it has an available charging station, and that the potential energy supply cannot exceed the product of charging power and dwell time. Constraints (10) and (11) specify the lower bound and upper bound of the battery power of an electric bus. Constraint (12) ensures that battery capacity cannot be negative.

By eliminating variables, $e_{k, i}^{\text {arriving }}$ and $s_{k, i}$, model $\mathrm{S} 1$ can be equivalently reformulated as a more concise program as 


$$
\begin{aligned}
& (\mathrm{S} 2): \min _{\left(\boldsymbol{x}, \boldsymbol{y}, \boldsymbol{e}^{\text {max }}, \boldsymbol{e}^{\text {leaving }}\right)} \sum_{k \in K} a^{\text {battery }} \zeta_{k} e_{k}^{\max } \\
& +\sum_{j \in \tilde{N}} \sum_{t \in T} a_{t}^{\text {charger }} x_{j, t}+\sum_{k \in K} \sum_{j \in N \backslash \tilde{N}} \sum_{t \in T} a_{t}^{\text {charger }} y_{k, j, t}
\end{aligned}
$$

s.t. (1)-(5), (11)-(12)

$$
\begin{gathered}
e_{k, j}^{\text {leaving }} \leq e_{k, i}^{\text {leaving }}-c_{k, i, j}+\sum_{t \in T} p_{t} x_{j, t} \tau_{k, j} \\
\forall k \in K, j \in N_{k} \cap \tilde{N},(i, j) \in L_{k} \\
e_{k, j}^{\text {leaving }} \leq e_{k, i}^{\text {leaving }}-c_{k, i, j}+\sum_{t \in T} p_{t} y_{k, j, t} \tau_{k, j} \\
\forall k \in K, j \in N_{k} \backslash \tilde{N},(i, j) \in L_{k} \\
e_{k, i}^{\text {leaving }}-c_{k, i, j} \geq \epsilon^{\text {lower }} e_{k}^{\max } \quad \forall k \in K,(i, j) \in L_{k}
\end{gathered}
$$

\section{Robust Formulation}

As formulated, the above deterministic optimization model is a mixed integer linear programming problem. In this section, we apply the set-based RO methodology to address the uncertainty in energy consumption of BEBs and to identify a robust solution for the planning problem of a fast-charging BEB system.

\section{Energy Consumption Uncertainty}

Under the set-based RO approach, it is assumed that energy consumption $c_{k, i, j}$ is unknown and belongs to a specified uncertainty set. Specifically, the following box uncertainty set is commonly used:

$$
c_{k, i, j} \in U_{k}^{b} \equiv\left[\bar{c}_{k, i, j}, \bar{c}_{k, i, j}+\hat{c}_{k, i, j} \sigma_{k, i, j}\right]
$$

where $\sigma_{k, i, j}$ is the uncertainty level coefficient; $\bar{c}_{k, i, j}$ and $\hat{c}_{k, i, j}$ are the nominal value and maximum deviation of energy consumption of a BEB from link $k \in K$ on link $(i, j) \in L_{k}$, respectively. In practice, both $\bar{c}_{k, i, j}$ and $\hat{c}_{k, i, j}$ can be calculated based on collected bus driving profile and ridership data. Here, possible negative deviations in energy consumption are neglected, because they are not adverse factors for the bus system.

The box uncertainty set only restricts the maximum deviation of each individual parameter $c_{k, i, j}$. Under the box uncertainty set, all energy consumption parameters can reach their worst-case value (i.e., the maximum value) simultaneously. In practice, however, it is very unlikely that there are heavy passenger demands and extremely bad traffic conditions in all parts of the bus route. Therefore, to find a less conservative solution, we impose $\Gamma_{k}$ as a budget for uncertainty to set an upper limit for the cumulative deviation in energy consumption along a route. Thus, the following polyhedral uncertainty set is used to replace the box uncertainty set as

$$
\begin{gathered}
\boldsymbol{c}_{k} \in U_{k}^{p} \equiv\left\{\boldsymbol{c}_{k} \in \mathbb{R}^{\left|L_{k}\right|} \mid \bar{c}_{k, i, j} \leq c_{k, i, j} \leq \bar{c}_{k, i, j}+\hat{c}_{k, i, j} \sigma_{k, i, j},\right. \\
\left.\sum_{(i, j) \in L_{k}}\left(c_{k, i, j}-\bar{c}_{k, i, j}\right) \leq \Gamma_{k}\right\}
\end{gathered}
$$

where $\boldsymbol{c}_{k}$ denotes the vector of $\left\{\cdots, c_{k, i, j}, \cdots\right\}$.

\section{Affinely Adjustable Robust Counterpart}

In the deterministic model S2, if constraints (13)-(15) must be satisfied for all realizations of $\boldsymbol{c}_{k} \in U_{k}^{p}$, then the corresponding solution is deemed robust against the uncertainty set $U_{k}^{p}$. A traditional set-based RO approach focuses on static problems and has an underlying assumption that all decision variables represent "here and now" decisions and therefore should be assigned specific numerical values as a result of solving the problem before the actual data "reveals itself" (10). In our problem, however, the change in battery power for a BEB depends on the actual realizations of uncertain energy consumption and should be a dynamic process. To consider such a dynamic nature, we adopt a more advanced approach of an affinely adjustable robust counterpart (AARC), as developed by Ben-Tal et al. (9). AARC allows some of the decision variables, including auxiliary variables (e.g., slack or surplus variables) and variables representing "wait and see" decisions (i.e., decisions that can be made when part of the uncertain data becomes known) (9), to be adjustable based on different realizations of uncertain data by introducing functional relationships between these decision variables and uncertain data. Moreover, by restricting the functional relationships to the affine, the AARC approach ensures tractable robust formulations.

For our problem, the decision variables include $x_{j, t}, y_{k, j, t}, e_{k}^{\max }$, and $e_{k, i}^{\text {leaving }}$, where $x_{j, t}$ and $y_{k, j, t}$ determine the deployment of fast-charging stations, $e_{k}^{\max }$ determines on-board battery sizes, and $e_{k, i}^{\text {leaving }}$ represents the remaining battery power of an electric bus at a certain station. From the perspective of planning, the deployment of fast-charging stations and the battery sizes of electric buses should be determined before construction of a fast-charging electric bus system. Thus, decision variables $x_{i, t}, y_{k, i, t}$, and $e_{k}^{\max }$ should represent "here and now" decisions and should not be adjustable variables. However, because the remaining battery power of an electric bus at a certain station is directly affected by actual energy consumption on those links that have been passed, variable $e_{k, i}^{\text {leaving }}$ should represent a "wait and see" decision (its value can be determined only when the actual energy consumption data on the links that have been passed "reveals itself"). Therefore, variable $e_{k, i}^{\text {leaving }}$ should be an adjustable variable. 
To derive the AARC of our problem, we should first define a functional relationship between the adjustable variable $e_{k, i}^{\text {leaving }}$ and uncertain energy consumption data. As discussed above, the value of $e_{k, i}^{\text {leaving }}$ depends on uncertain energy consumption data on those links that have been passed. Let $L_{k}^{i}$ denote the set of links that an electric bus needs to pass from the base station to station $i \in N_{k}$ along the route of bus line $k \in K$. Note that for the base station of a bus line $i=o_{k}$, we set $L_{k}^{i}=L_{k}$. Let $c_{k}^{i}$ denote the vector of $\left\{\cdots, c_{k, m, n}, \cdots\right\}$, where $(m, n) \in L_{k}^{i}$. The adjustable variable $e_{k, i}^{\text {leaving }}$ can then be represented as the affine function

$$
e_{k, i}^{\text {leaving }}\left(\boldsymbol{c}_{k}^{i}\right)=\theta_{k}^{i}+\sum_{(m, n) \in L_{k}^{i}} \lambda_{k, m, n}^{i} c_{k, m, n}
$$

where $\theta_{k}^{i}$ and $\lambda_{k, m, n}^{i}$ are new decision variables that are not adjustable.

Substituting the adjustable variable, we arrive at the AARC formulation

$$
\begin{aligned}
& (\mathrm{S} 2-\mathrm{AARC}): \min _{\left(\boldsymbol{x}, \boldsymbol{y}, \boldsymbol{e}^{\max }, \boldsymbol{\theta}, \boldsymbol{\lambda}\right)} \sum_{k \in K} a^{\text {battery }} \zeta_{k} e_{k}^{\max } \\
& +\sum_{j \in \tilde{N}} \sum_{t \in T} a_{t}^{\text {charger }} x_{j, t}+\sum_{k \in K} \sum_{j \in N \backslash \tilde{N}} \sum_{t \in T} a_{t}^{\text {charger }} y_{k, j, t}
\end{aligned}
$$

s.t. (1)-(4), (12)

$$
\begin{gathered}
\theta_{k}^{i}+\sum_{(m, n) \in L_{k}^{i}} \lambda_{k, m, n}^{i} c_{k, m, n}=\epsilon^{\mathrm{upper}} e_{k}^{\max } \\
\forall k \in K, i=o_{k}, \boldsymbol{c}_{k} \in U_{k}^{p} \\
\theta_{k}^{j}+\sum_{(m, n) \in L_{k}^{j}} \lambda_{k, m, n}^{j} c_{k, m, n} \leq \theta_{k}^{i}+\sum_{(m, n) \in L_{k}^{i}} \lambda_{k, m, n}^{i} c_{k, m, n}-c_{k, i, j} \\
+\sum_{t \in T} p_{t} x_{j, t} \tau_{k, j} \quad \forall k \in K, j \in N_{k} \cap \tilde{N},(i, j) \in L_{k}, \boldsymbol{c}_{k} \in U_{k}^{p}
\end{gathered}
$$$$
\theta_{k}^{j}+\sum_{(m, n) \in L_{k}^{j}} \lambda_{k, m, n}^{j} c_{k, m, n} \leq \theta_{k}^{i}+\sum_{(m, n) \in L_{k}^{i}} \lambda_{k, m, n}^{i} c_{k, m, n}-c_{k, i, j}
$$$$
+\sum_{t \in T} p_{t} y_{k, j, t} \tau_{k, j} \quad \forall k \in K, j \in N_{k} \backslash \tilde{N},(i, j) \in L_{k}, \boldsymbol{c}_{k} \in U_{k}^{p}
$$$$
\theta_{k}^{i}+\sum_{(m, n) \in L_{k}^{i}} \lambda_{k, m, n}^{i} c_{k, m, n}-c_{k, i, j} \geq \epsilon^{\mathrm{lower}} e_{k}^{\max }
$$$$
\forall k \in K,(i, j) \in L_{k}, c_{k} \in U_{k}^{p}
$$$$
\theta_{k}^{i}+\sum_{(m, n) \in L_{k}^{i}} \lambda_{k, m, n}^{i} c_{k, m, n} \leq \epsilon^{\mathrm{upper}} e_{k}^{\max }
$$$$
\forall k \in K, i \in N_{k}, \boldsymbol{c}_{k} \in U_{k}^{p}
$$

In S2-AARC, the adjustable variable $e_{k, i}^{\text {leaving }}$ is no longer a decision variable, but the coefficients of the affine function of $e_{k, i}^{\text {leaving }}$, including $\theta_{k}^{i}$ and $\lambda_{k, m, n}^{i}$, become new decision variables. This means that the solution of S2AARC only determines the relationship between $e_{k, i}^{\text {leaving }}$ and uncertain energy consumption. Specific values of $e_{k, i}^{\text {leaving }}$ can be calculated following the realization of energy consumption.

\section{Tractable Reformulation of S2-AARC}

In the above formulation, we observe that each piece of constraint in constraints (16)-(20) (i.e., for a certain combination of superscripts and subscripts) actually consists of a continuum, or an infinite number, of constraints, because the formulation must be satisfied for all realizations of uncertain energy consumption. Therefore, S2AARC is a semi-infinite programming problem (the number of variables is finite) that is computationally intractable. As shown in Proposition 1, we can further reformulate S2-AARC as a tractable optimization problem.

Proposition 1. Given the polyhedral uncertainty set, $U_{k}^{p}$, S2-AARC can be equivalently reformulated as the mixed integer linear programming problem

$$
\begin{aligned}
& (\mathrm{S} 2-\mathrm{AARC}-\mathrm{T}): \min _{\left(\boldsymbol{x}, \boldsymbol{y}, \boldsymbol{e}^{\max }, \boldsymbol{\theta}, \boldsymbol{\lambda}, \boldsymbol{\omega}\right)} \sum_{k \in K} a^{\text {battery }} \zeta_{k} e_{k}^{\max } \\
& +\sum_{j \in \tilde{N}} \sum_{t \in T} a_{t}^{\text {charger }} x_{j, t}+\sum_{k \in K} \sum_{j \in N \backslash \tilde{N}} \sum_{t \in T} a_{t}^{\text {charger }} y_{k, j, t}
\end{aligned}
$$

s.t. (1)-(4), (12)

$$
\begin{gathered}
\theta_{k}^{i}=\epsilon^{\mathrm{upper}} e_{k}^{\max } \quad \forall k \in K, i=o_{k} \\
\lambda_{k, m, n}^{i}=0 \quad \forall k \in K, i=o_{k},(m, n) \in L_{k}^{i} \\
\sum_{(m, n) \in L_{k}}\left(\bar{c}_{k, i, j}+\hat{c}_{k, i, j} \sigma_{k, i, j}\right) \omega_{k m n}^{i 1}-\sum_{(m, n) \in L_{k}} \bar{c}_{k, m, n} \omega_{k m n}^{i 2} \\
+\left(\Gamma_{k}+\sum_{(m, n) \in L_{k}} \bar{c}_{k, m, n}\right) \omega_{k}^{i 3} \leq \theta_{k}^{i}-\theta_{k}^{j} \\
+\sum_{t \in T} p_{t} x_{j, t} \tau_{k, j} \quad \forall k \in K, j \in N_{k} \cap \tilde{N},(i, j) \in L_{k} \\
\omega_{k m n}^{i 1}-\omega_{k m n}^{i 2}+\omega_{k}^{i 3}=\lambda_{k, m, n}^{j}-\lambda_{k, m, n}^{i} \\
\forall k \in K, j \in N_{k} \cap \tilde{N},(i, j) \in L_{k},(m, n) \in L_{k}^{i} \\
\omega_{k i j}^{i 1}-\omega_{k i j}^{i 2}+\omega_{k}^{i 3}=\lambda_{k, i, j}^{j}+1 \\
\forall k \in K, j \in N_{k} \cap \tilde{N},(i, j) \in L_{k} \\
\omega_{k m n}^{i 1}-\omega_{k m n}^{i 2}+\omega_{k}^{i 3}=0 \\
\forall k \in K, j \in N_{k} \cap \tilde{N},(i, j) \in L_{k},(m, n) \in L_{k} \backslash L_{k}^{j}
\end{gathered}
$$




$$
\begin{aligned}
& \omega_{k m n}^{i 1}, \omega_{k m n}^{i 2}, \omega_{k}^{i 3} \geq 0 \\
& \forall k \in K, j \in N_{k} \cap \tilde{N},(i, j) \in L_{k},(m, n) \in L_{k} \\
& \sum_{(m, n) \in L_{k}}\left(\bar{c}_{k, i, j}+\hat{c}_{k, i, j} \sigma_{k, i, j}\right) \omega_{k m n}^{i 4}-\sum_{(m, n) \in L_{k}} \bar{c}_{k, m, n} \omega_{k m n}^{i 5} \\
& +\left(\Gamma_{k}+\sum_{(m, n) \in L_{k}} \bar{c}_{k, m, n}\right) \omega_{k}^{i 6} \leq \theta_{k}^{i}-\theta_{k}^{j} \\
& +\sum_{t \in T} p_{t} y_{k, j, t} \tau_{k, j} \quad \forall k \in K, j \in N_{k} \backslash \tilde{N},(i, j) \in L_{k} \\
& \omega_{k m n}^{i 4}-\omega_{k m n}^{i 5}+\omega_{k}^{i 6}=\lambda_{k, m, n}^{j}-\lambda_{k, m, n}^{i} \\
& \forall k \in K, j \in N_{k} \backslash \tilde{N},(i, j) \in L_{k},(m, n) \in L_{k}^{i} \\
& \omega_{k m n}^{i 4}-\omega_{k m n}^{i 5}+\omega_{k}^{i 6}=\lambda_{k, i, j}^{j}+1 \\
& \forall k \in K, j \in N_{k} \backslash \tilde{N},(i, j) \in L_{k} \\
& \omega_{k m n}^{i 4}-\omega_{k m n}^{i 5}+\omega_{k}^{i 6}=0 \\
& \forall k \in K, j \in N_{k} \backslash \tilde{N},(i, j) \in L_{k},(m, n) \in L_{k} \backslash L_{k}^{j} \\
& \omega_{k m n}^{i 4}, \omega_{k m n}^{i 5}, \omega_{k}^{i 6} \geq 0 \\
& \forall k \in K, j \in N_{k} \backslash \tilde{N},(i, j) \in L_{k},(m, n) \in L_{k} \\
& \sum_{(m, n) \in L_{k}}\left(\bar{c}_{k, i, j}+\hat{c}_{k, i, j} \sigma_{k, i, j}\right) \omega_{k m n}^{i 7}-\sum_{(m, n) \in L_{k}} \bar{c}_{k, m, n} \omega_{k m n}^{i 8} \\
& +\left(\Gamma_{k}+\sum_{(m, n) \in L_{k}} \bar{c}_{k, m, n}\right) \omega_{k}^{i 9} \leq \theta_{k}^{i}-\epsilon^{\text {lower }} e_{k}^{\max } \\
& \forall k \in K,(i, j) \in L_{k} \\
& \omega_{k m n}^{i 7}-\omega_{k m n}^{i 8}+\omega_{k}^{i 9}=-\lambda_{k, m, n}^{i} \\
& \forall k \in K, i \in N_{k},(i, j) \in L_{k},(m, n) \in L_{k}^{i} \\
& \omega_{k m n}^{i 7}-\omega_{k m n}^{i 8}+\omega_{k}^{i 9}=1 \\
& \forall k \in K, i \in N_{k},(i, j) \in L_{k} \\
& \omega_{k m n}^{i 7}-\omega_{k m n}^{i 8}+\omega_{k}^{i 9}=0 \\
& \forall k \in K, i \in N_{k},(i, j) \in L_{k},(m, n) \in L_{k} \backslash L_{k}^{j} \\
& \omega_{k m n}^{i 7}, \omega_{k m n}^{i 8}, \omega_{k}^{i 9} \geq 0 \quad \forall k \in K, i \in N_{k},(i, j) \in L_{k},(m, n) \in L_{k} \\
& \sum_{(m, n) \in L_{k}}\left(\bar{c}_{k, i, j}+\hat{c}_{k, i, j} \sigma_{k, i, j}\right) \omega_{k m n}^{i 10}-\sum_{(m, n) \in L_{k}} \bar{c}_{k, m, n} \omega_{k m n}^{i 11} \\
& +\left(\Gamma_{k}+\sum_{(m, n) \in L_{k}} \bar{c}_{k, m, n}\right) \omega_{k}^{i 12} \leq \epsilon^{\mathrm{upper}} e_{k}^{\max }-\theta_{k}^{i} \\
& \forall k \in K, i \in N_{k}
\end{aligned}
$$

$$
\begin{gathered}
\omega_{k m n}^{i 10}-\omega_{k m n}^{i 11}+\omega_{k}^{i 12}=\lambda_{k, m, n}^{i} \quad \forall k \in K, i \in N_{k},(m, n) \in L_{k}^{i} \\
\omega_{k m n}^{i 10}-\omega_{k m n}^{i 11}+\omega_{k}^{i 12}=0 \quad \forall k \in K, i \in N_{k},(m, n) \in L_{k} \backslash L_{k}^{i} \\
\omega_{k m n}^{i 10}, \omega_{k m n}^{i 11}, \omega_{k}^{i 12} \geq 0 \quad \forall k \in K, i \in N_{k},(m, n) \in L_{k} \backslash L_{k}^{i}
\end{gathered}
$$

where $\omega$ is a set of dual variables. Note that the numerical superscripts are used for simplicity of notation.

Proof. For constraint (16) in S2-AARC, because it must be satisfied for all realizations of uncertain energy consumption $\boldsymbol{c}_{k} \in U_{k}^{p}$, it is straightforward to prove its equivalency with constraints (21) and (22). Except for constraint (16), each constraint affected by energy consumption uncertainty in S2-AARC (i.e., each of constraints (17)-(20)) can be generalized by

$$
\sum_{(m, n) \in L_{k}} \alpha_{k, m, n}^{i} c_{k, m, n} \leq h^{i} \quad \forall c_{k} \in U_{k}^{p}
$$

For instance, for constraint (17), $\alpha_{k, m, n}^{i}=\lambda_{k, m, n}^{j}-\lambda_{k, m, n}^{i}$ for $\quad \forall(m, n) \in L_{k}^{i}, \quad \alpha_{k, i, j}^{i}=\lambda_{k, i, j}^{j}+1, \quad \alpha_{k, m, n}^{i}=0 \quad$ for $\forall(m, n) \in L_{k} \backslash L_{k}^{j}$, and $h^{i}=\theta_{k}^{i}-\theta_{k}^{j}+\sum_{t \in T} p_{t} x_{j, t} \tau_{k, j}$. Note that constraints (17)-(19) are link-based, and superscript $i$ in constraint (42) corresponds to the starting node of link $(i, j) \in L_{k}$.

Constraint (42) can be equivalently given as

$$
\max _{\boldsymbol{c}_{k} \in U_{k}^{p}} \sum_{(m, n) \in L_{k}} \alpha_{k, m, n}^{i} c_{k, m, n} \leq h^{i}
$$

Without loss of generality, $U_{k}^{p}=\left\{\boldsymbol{c}_{k} \in \mathbb{R}^{\left|L_{k}\right|} \mid \bar{c}_{k, i, j}\right.$ $\left.\leq c_{k, i, j} \leq \bar{c}_{k, i, j}+\hat{c}_{k, i, j} \sigma_{k, i, j}, \sum_{(i, j) \in L_{k}}\left(c_{k, i, j}-\bar{c}_{k, i, j}\right) \leq \Gamma_{k}\right\}$ can be represented as $A \boldsymbol{c}_{k} \leq \boldsymbol{b}$, and $\max _{\boldsymbol{c}_{k} \in U_{k}^{p}} \sum_{(m, n) \in L_{k}} \alpha_{k, m, n}^{i} c_{k, m, n}$ can be written as the optimization problem

$$
(P): \max _{\boldsymbol{c}_{k}} \boldsymbol{\alpha}_{\boldsymbol{k}}^{i} \boldsymbol{c}_{\boldsymbol{k}}
$$

s.t.

$$
\begin{aligned}
& A \boldsymbol{c}_{\boldsymbol{k}} \leq \boldsymbol{b} \\
& \boldsymbol{c}_{\boldsymbol{k}} \geq 0
\end{aligned}
$$

According to the property of strong duality $(10,16)$, the equivalent dual problem (D) of $(\mathrm{P})$ is given as 


$$
\text { (D) : } \min _{\boldsymbol{\omega}} \boldsymbol{b \omega}
$$

s.t.

$$
\begin{aligned}
& A^{T} \boldsymbol{\omega}=\boldsymbol{\alpha}_{\boldsymbol{k}}^{\boldsymbol{i}} \\
& \boldsymbol{\omega} \geq 0
\end{aligned}
$$

where $\boldsymbol{\omega}$ is a vector of dual variables.

Because the programming of (D) is equivalent to that of $(\mathrm{P})$, they will have the same optimal value. Therefore, constraint (43), which requires that the optimal value of $(\mathrm{P}), \leq h^{i}$, is equivalent to the optimal value of $(\mathrm{D})$, is achieved and is $\leq h^{i}$. Because (D) is a minimization problem, the requirement that the optimal value of (D) is $\leq h^{i}$ is equivalent to the requirement that (D) has a feasible solution $\boldsymbol{\omega}$ with $\boldsymbol{b} \boldsymbol{\omega} \leq h^{i}$. Therefore, constraint (43) can be equivalently replaced by $\boldsymbol{b} \boldsymbol{\omega} \leq h^{i}, A^{T} \boldsymbol{\omega}=\boldsymbol{\alpha}_{\boldsymbol{k}}^{i}$ and $\boldsymbol{\omega} \geq 0$, and the reformulation of S2-AARC-T becomes a mixed integer linear programming problem, which is tractable.

\section{Numerical Study}

To demonstrate the effectiveness of the proposed model, a numerical study is presented. The case study is based on the bus system in downtown Salt Lake City, Utah, in the United States.

\section{Bus System and Model Parameters}

A subnetwork of the Salt Lake City bus system, as shown in Figure 1, is considered in the case study. The subnetwork covers 91.4 kilometers of road segments and includes eight bus lines, with a total of 34 buses serving 315 bus stations. Assuming that the transit agency wants to transform this bus system into a fast-charging electric bus system, the locations and types of charging stations, as well as the battery capacity of the electric buses, must be determined.

To evaluate the energy consumption of an electric bus on each link for each bus line, an energy consumption model proposed by Wang et al. (17) has been adopted. The model is derived from the analysis of forces exerted on a running electric bus, which considers air resistance, rolling resistance, climbing resistance, acceleration resistance and regenerative braking. Table $2 a$ reveals the simulation parameters applied to calculate energy consumption. For simplicity, it is assumed that all roads in the network have the same friction factor, and that the eight bus lines use the same type of electric buses. The slope profile is calculated based on Digital Elevation Model (DEM) data from the Utah Automated Geographic Reference Center (AGRC). The driving profiles are extracted from live Service Interface for Real Time Information (SIRI) vehicle monitoring data, which

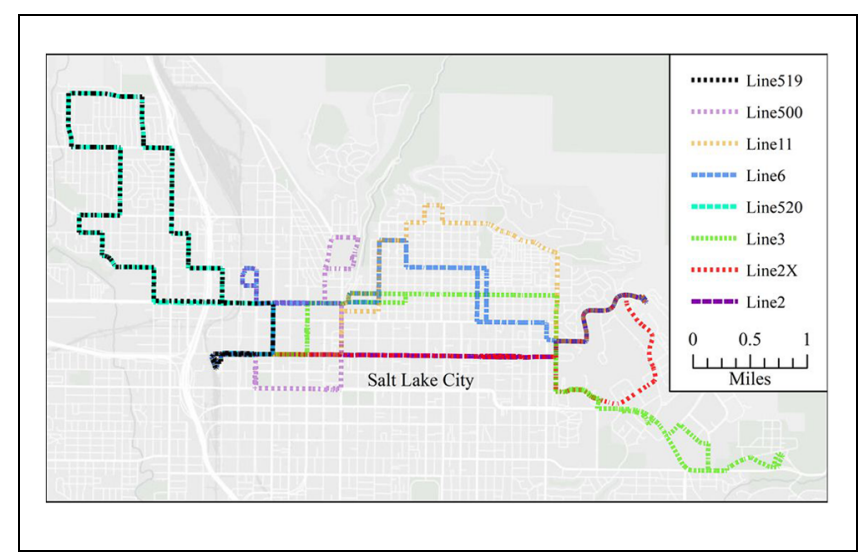

Figure I. Salt Lake City bus system.

is collected using the "VehicleMonitoring by Route" service implemented by the Utah Transit Authority (UTA) (18). The data was collected from October 10, 2017, to November 5, 2017. In total, 1,086, 216, 510, 608, 324, 486, 590 and 414 driving loop profiles for bus lines 2, $2 \mathrm{X}, 3,6,11,500,519$ and 520, respectively were obtained. Figure $2 a$ lists some sample data for bus line 2 , which corresponds to two service loops of a vehicle on

\begin{tabular}{|c|c|}
\hline & Value \\
\hline \multicolumn{2}{|l|}{$\begin{array}{l}\text { Parameters of Energy Consumption Model } \\
\text { Parameter }\end{array}$} \\
\hline Friction factor & 0.02 \\
\hline Total mass of a bus $(\mathrm{Kg})$ & 20,400 \\
\hline Gravity acceleration $\left(\mathrm{m} / \mathrm{s}^{2}\right)$ & 9.81 \\
\hline Air density $\left(\mathrm{kg} / \mathrm{m}^{3}\right)$ & 1.2 \\
\hline Air resistance coefficient & 0.7 \\
\hline Bus frontal area $\left(\mathrm{m}^{2}\right)$ & 7.5 \\
\hline Energy output efficiency & $60 \%$ \\
\hline Energy input efficiency & $50 \%$ \\
\hline \multicolumn{2}{|l|}{$\begin{array}{l}\text { Parameters of Batteries and Charging Stations } \\
\text { Parameter }\end{array}$} \\
\hline $\begin{array}{l}\text { Charging power of a type I charging station } \\
(\mathrm{kW})\end{array}$ & 90 \\
\hline $\begin{array}{l}\text { Charging power of a type } 2 \text { charging station } \\
(\mathrm{kW})\end{array}$ & 250 \\
\hline $\begin{array}{l}\text { Amortized cost of installing and maintaining a } \\
\text { type I charging station }(\$)\end{array}$ & 4,637 \\
\hline $\begin{array}{l}\text { Amortized cost of installing and maintaining a } \\
\text { type } 2 \text { charging station }(\$)\end{array}$ & 11,589 \\
\hline $\begin{array}{l}\text { Amortized cost of battery, including } \\
\text { replacement }(\$ / \mathrm{kWh})\end{array}$ & 148 \\
\hline $\begin{array}{l}\text { Lower bound of remaining battery power (in } \\
\text { percentage of battery capacity) }\end{array}$ & 0.3 \\
\hline $\begin{array}{l}\text { Upper bound of remaining battery power (in } \\
\text { percentage of battery capacity) }\end{array}$ & 0.8 \\
\hline \multicolumn{2}{|l|}{ Parameters of Uncertainty Set } \\
\hline Uncertainty level of box uncertainty set & $1.0,2.0,3.0$ \\
\hline Uncertainty level of budget uncertainty set & $0-1.0$ \\
\hline
\end{tabular}
October 12. The dwelling time data can be extracted

Table 2. Model Parameters 


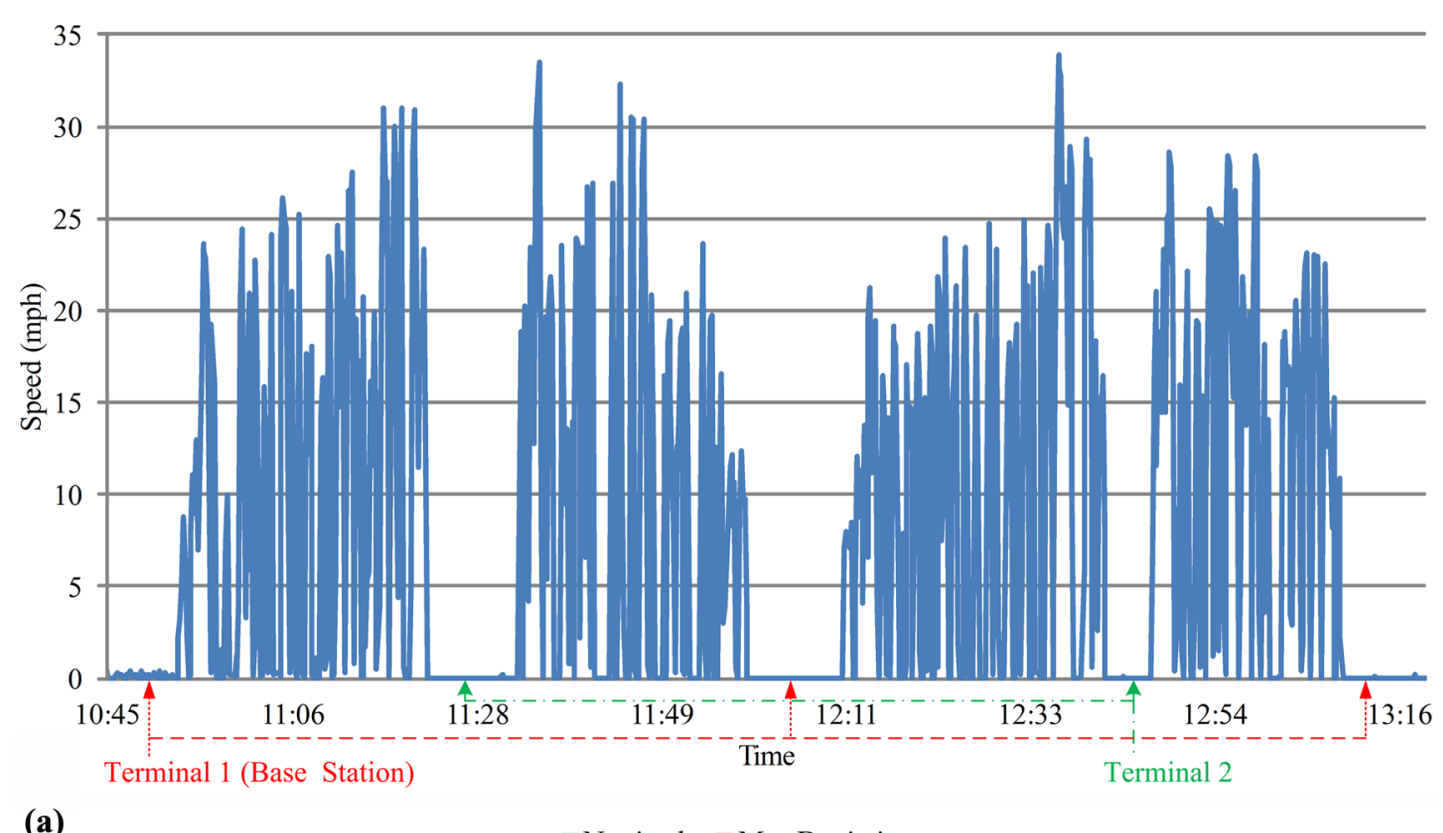

(a)

- Nonimal $\quad$ Max Deviation

$$
3000
$$

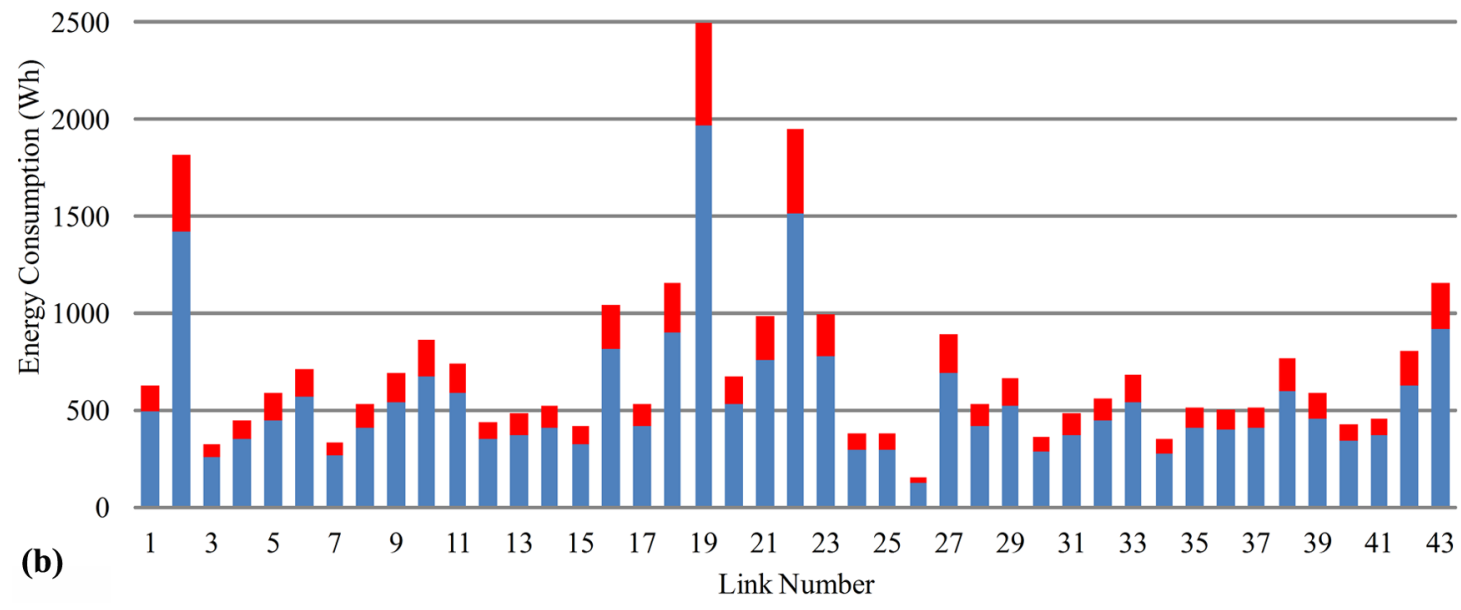

Figure 2. (a) Sample speed profile data for line 2, and (b) energy consumption data for line 2.

from the driving profiles. The ridership data was requested from the UTA. The average weight of a passenger is assumed to be $70 \mathrm{~kg}$. Based on the collected data, the energy consumption data can then be calculated. Figure $2 b$ reveals the energy consumption data for bus line 2 .

The parameters relating to batteries and fast-charging stations are given in Table $2 b$. For simplicity, we only consider two types of fast-charging stations, whose charging power and costs are $90 \mathrm{~kW}, \$ 50,000$ (19) and $250 \mathrm{~kW}, \$ 125,000$ (20), respectively. We further assume that the service life of charging stations is 12 years, and that the maintenance cost is $1 \%$ of the installation cost per year. For the on-board batteries, we consider the lithium-titanate battery (referred to as LTO in the battery industry) utilized in Proterra's battery electric CATALYST Fast Charge transit bus. The battery has a service life of six years with a cost of around $\$ 1000 / \mathrm{kWh}$ (21). Considering a 0.01 discount rate, we can then calculate the amortized cost of charging stations and batteries.

The uncertainty set in our robust model is given by $\boldsymbol{c}_{\boldsymbol{k}} \in U_{k}^{p} \equiv\left\{\boldsymbol{c}_{\boldsymbol{k}} \in \mathbb{R}^{\left|L_{k}\right|} \mid \bar{c}_{k, i, j} \leq c_{k, i, j} \leq \bar{c}_{k, i, j}+\hat{c}_{k, i, j} \sigma_{k, i, j}\right.$, 
$\left.\sum_{(i, j) \in L_{k}}\left(c_{k, i, j}-\bar{c}_{k, i, j}\right) \leq \Gamma_{k}\right\} \cdot \bar{c}_{k, i, j}$ and $\hat{c}_{k, i, j}$ are calculated based on real-world data. Parameters $\sigma_{k, i, j}$ and $\Gamma_{k}$ represent the conservation level of the robust model and are determined in practice by decision makers or planners. In this numerical study, we assume that $\sigma_{k, i, j}$ (representing the conservation level of the box uncertainty set) is uniform for all bus lines and all links and considers three conservation levels: $\sigma_{k, i, j} \in\{1.0,2.0,3.0\}$. For the budget of uncertainty, we use the ratio of the uncertainty budget to the total possible energy consumption deviation along a route (i.e., $\Gamma^{k} / \sum_{(i, j) \in L_{k}} \hat{c}_{k, i, j} \sigma_{k, i, j}$ ), denoted as $\gamma^{k}$, to represent the conservation level. We further assume that $\gamma^{k}$ is identical for all bus lines and consider 11 levels of uncertainty with $\gamma^{k}$ ranging between 0 and 1 , with a step size of 0.1 .

\section{Results and Analysis}

Based on the network of the Salt Lake City bus system, we obtain a robust model with 171,951 variables (644 binary variables) and 86,217 constraints. We solve this model using GAMS (22) with a CPLEX (23) solver on a $3.40 \mathrm{GHz}$ Dell Computer with $16 \mathrm{~GB}$ of RAM. With a $0.1 \%$ relative optimality gap, the computation time of robust models ranges from 10 to 60 minutes, depending on the uncertainty level. Note that when $\gamma^{k}$ is zero, the robust model will have the same solution as the deterministic model. Table 3 lists the comparison between the results of one robust model with an uncertainty level of $\sigma_{k, i, j}=2.0$ and $\gamma^{k}=1.0$ and the results of the corresponding deterministic model. Compared to the deterministic model, the robust model requires larger batteries for all eight bus lines. Moreover, as shown in Figure $3 a$, $b$, the deployment and the number of fast-charging stations in the robust model is also different from that of the deterministic model. We observe that when compared to the deterministic solution, the robust solution deploys more $250 \mathrm{~kW}$ fast-charging stations and less 90 $\mathrm{kW}$ fast-charging stations. In addition, bus lines 6, 11, and 500 use $90 \mathrm{~kW}$ fast-charging stations at the base station in the deterministic solution, whereas they require $250 \mathrm{~kW}$ fast-charging stations in the robust solution. When considering the uncertainty of energy consumption at the levels of $\sigma_{k, i, j}=2.0$ and $\gamma^{k}=1.0$, the amortized cost of the electric bus system will increase from $\$ 347,835$ to $\$ 481,277$ under the robust model.

Although the robust optimal solution requires greater investments, the corresponding electric bus system can operate uninterrupted when energy consumption experiences deviations within the uncertainty set. Consider a worst-case scenario in which all of the energy consumption $c_{k, i, j}$ has a deviation from the expected value $\bar{c}_{k, i, j}$ which is double that of the calculated maximum deviation $\hat{c}_{k, i, j}$. Under this scenario, the solutions of robust models with conservation levels of $\sigma_{k, i, j} \geq 2.0$ and $\gamma^{k} \geq 1.0$ will still be feasible, and the corresponding electric bus system can operate normally, whereas the solution of the deterministic model will become infeasible, and the corresponding electric bus system cannot satisfy the energy requirements for normal operations. Under the worst-case scenario, Figure 4 lists comparisons of the

Table 3. Comparison between the Deterministic Model and A Robust Model $\left(\sigma_{k, i, j}=2.0, \gamma^{k}=1.0\right)$

\begin{tabular}{llll}
\hline Battery size comparison & & & \\
& Battery capacity $(\mathrm{kWh})$ & Robust model & Battery size increase \\
Shuttle Line & Deterministic model & $35.1 \%$ \\
519 & 55.9 & 75.5 & $37.3 \%$ \\
520 & 55.2 & 75.8 & $90.0 \%$ \\
500 & 32.0 & 60.8 & $96.7 \%$ \\
11 & 33.2 & 65.3 & $113.6 \%$ \\
6 & 32.4 & 69.2 & $39.0 \%$ \\
3 & 59.2 & 82.3 & $62.7 \%$ \\
2 & 15.8 & 25.7 & $14.0 \%$ \\
$2 X$ & 25.0 & 28.5 & - \\
Comparison of fast-charging stations & & & \\
Items & Number & Robust model & \\
$90 \mathrm{~kW}$ station & Deterministic model & 6 & 15 \\
$250 \mathrm{~kW}$ station & 9 & & \\
Cost comparison & 11 & Robust model & Cost increase \\
Items & & $\$ 279,620$ & $56.5 \%$ \\
Battery & Cost & $\$ 201,657$ & $19.2 \%$ \\
Fast-charging stations & Deterministic model & $38.4 \%$ \\
Total & $\$ 178,623$ & $\$ 481,277$ &
\end{tabular}




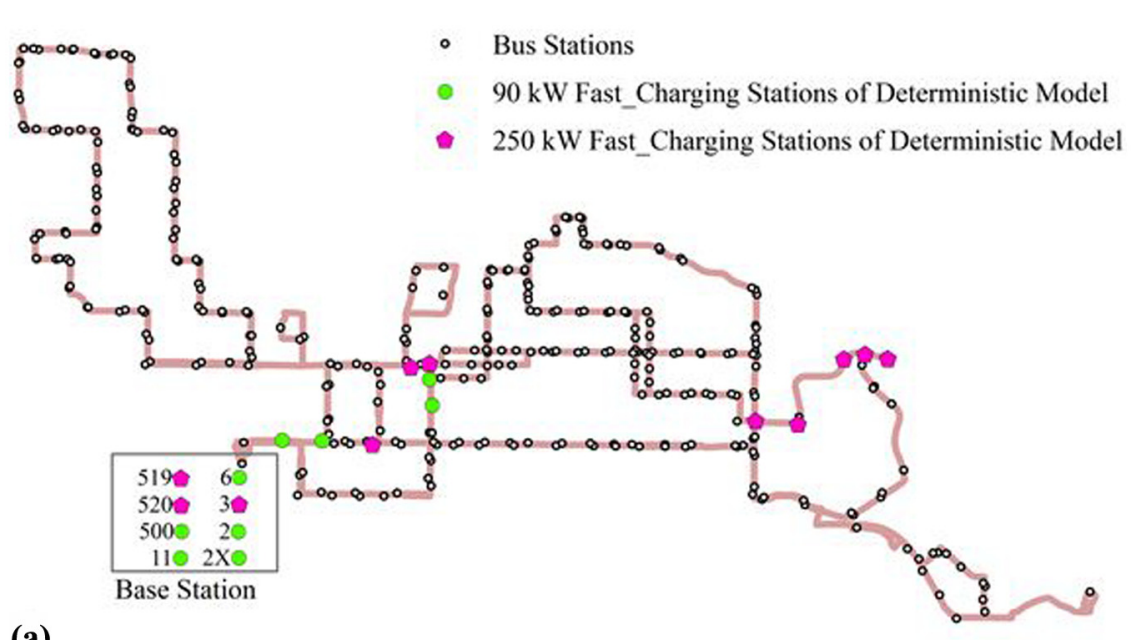

(a)

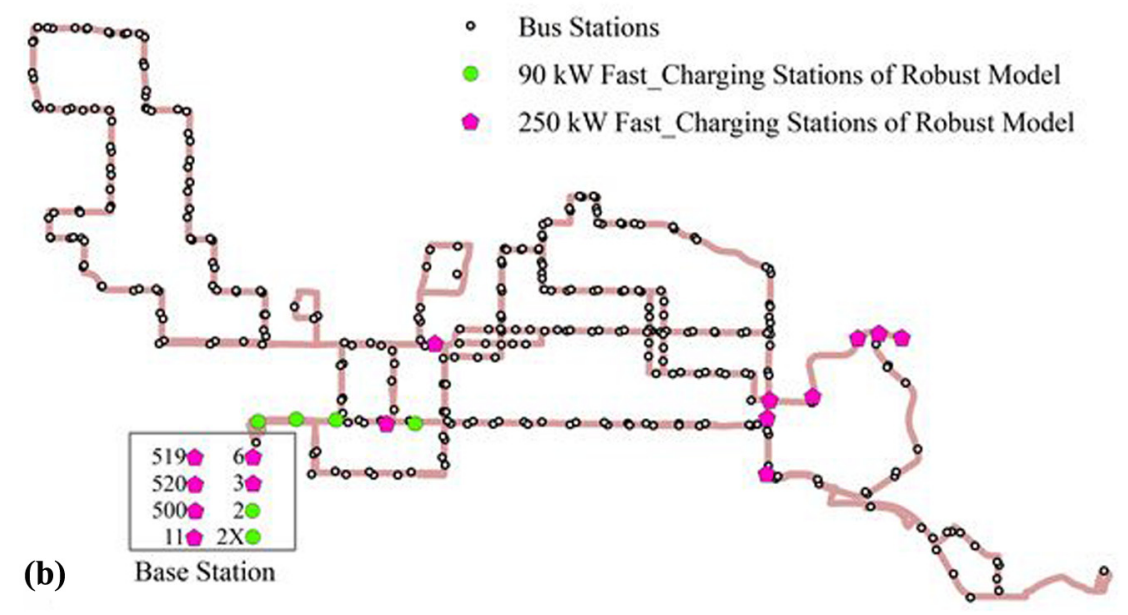

Figure 3. (a) Deployment of fast-charging stations in the deterministic model, and (b) deployment of fast-charging stations in a robust model.

battery level profiles of bus line 6 in its first service loop between the deterministic solution and the robust solution, with $\sigma_{k, i, j}=2.0$ and $\gamma^{k}=1.0$. We can observe that bus line 6 under the robust solution can operate normally within the given range of the battery level, whereas bus line 6 under the deterministic solution will run out of energy at the 63rd station and cannot return to the base station. Therefore, the performance of the robust solution is superior to that of the deterministic solution under the worst-case scenario.

With our robust model, we can obtain the optimal design for a fast-charging electric bus system that is robust against the uncertainty of energy consumption. However, additional investments will be required when an optimal robust design is sought. In practice, decision makers must determine the trade-off between robustness and the total cost of the bus system. For the bus system under study, Figure 5 compares the total amortized cost under different levels of conservation (or robustness). We can observe that under a certain box uncertainty level $\sigma_{k, i, j}$, the total cost increases as the budget uncertainty level $\gamma^{k}$ increases. Moreover, under a certain budget uncertainty level $\gamma^{k}>0$, a higher box uncertainty level leads to a higher total cost. This is expected under the robust model, because as the size of the uncertainty set for energy consumption increases, the electric bus system will require larger batteries, or a greater number of higher power fast-charging stations, or both, thus leading to a higher total cost. Under a certain box uncertainty level $\sigma_{k, i, j}$, the size of the polyhedral uncertainty set will increase as the budget uncertainty level $\gamma^{k}$ increases. Under a certain budget uncertainty level $\gamma^{k}>0$, the size of the polyhedral uncertainty set will increase as the box uncertainty level $\sigma_{k, i, j}$ increases.

\section{Conclusion}

In this study, we address the robust optimal planning problem of a fast-charging electric bus system considering 


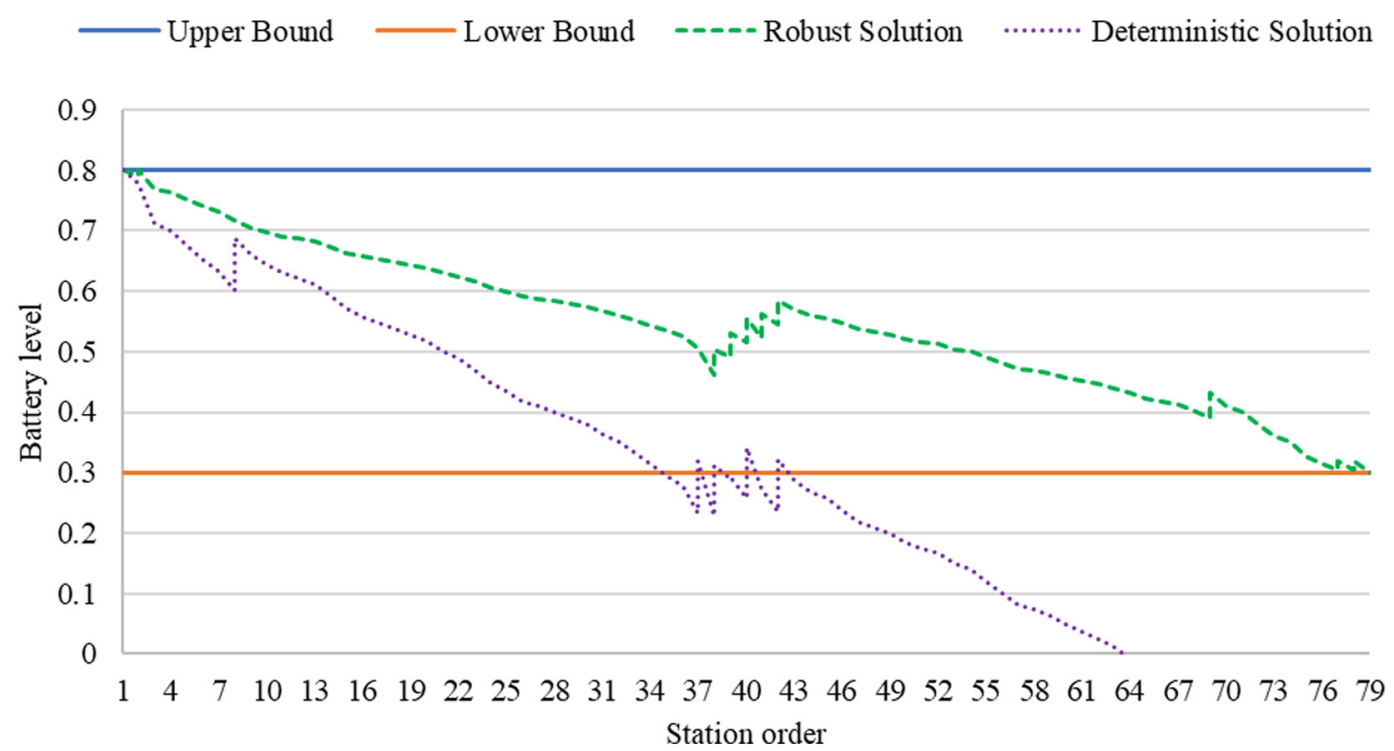

Figure 4. Comparison of battery level profiles of bus line 6 .

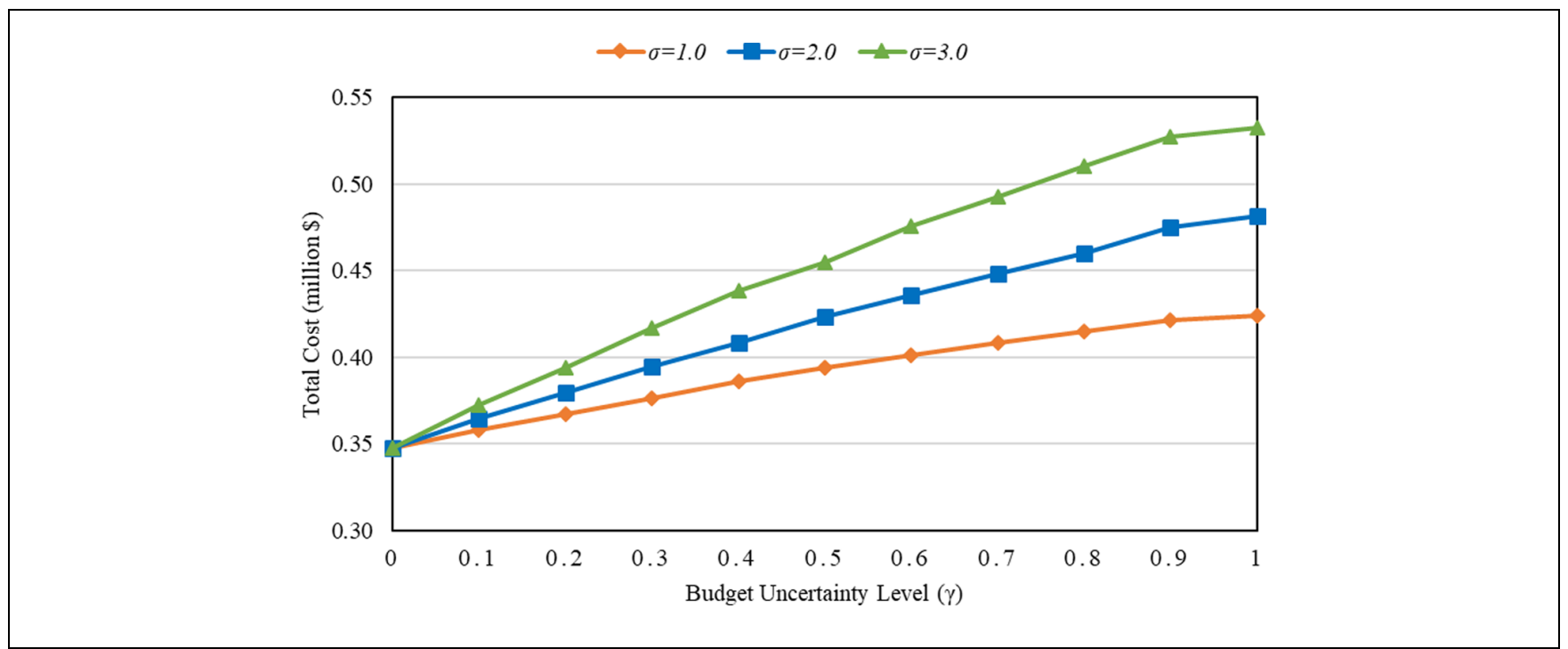

Figure 5. System cost under different uncertainty levels.

the uncertain energy consumption of buses. We first formulate a deterministic optimization model to determine the types and the deployment of fast-charging stations and the sizes of on-board batteries. Using the RO technique, we then derive the robust counterpart of the deterministic model. The uncertainty of energy consumption is bound by the intersection of the box uncertainty set and the budget uncertainty set. We apply the AARC approach to derive a less conservative and computationally tractable robust model. The model is tested with a real-world, large-scale numerical example. The results demonstrate that our model is able to solve the robust planning problem of a fast-charging electric bus system, and the optimal design is robust against uncertain energy consumption data. The comparison between the solutions of the deterministic model and those of the robust model under a worst-case scenario demonstrate that the robust model provides solutions that are robust against energy consumption uncertainty. Using different uncertainty levels, we investigate the relationship between the total cost and the level of robustness in a fast-charging electric bus system. The results may help decision-makers to determine the best 
trade-off between investments and the level of robustness in a fast-charging electric bus system.

The fast-charging electric bus system, as an emerging clean and sustainable alternative to a traditional diesel bus system, may be widely adopted in the near future. The proposed modeling framework in this study provides practitioners with an effective tool in the design of a robust optimal fast-charging electric bus system.

\section{Acknowledgments}

The study was partially sponsored by the U.S. Department of Energy (DE-EE0007997).

\section{Author Contributions}

The authors confirm contribution to the paper as follows: study conception and design: Zhaocai Liu, Ziqi Song, Yi He; data collection: Yi He; analysis and interpretation of results: Zhaocai Liu, Ziqi Song, Yi He; draft manuscript preparation: Zhaocai Liu. All authors reviewed the results and approved the final version of the manuscript.

\section{References}

1. Chen, J., B. Atasoy, T. Robenek, M. Bierlaire, and M. Thémans. Planning of Feeding Station Installment for Electric Urban Public Mass-transportation System. Proc., 13th Swiss Transportation Research Conference, 2013, No. EPFL-CONF-195881.

2. Liu, Z., Z. Song, and Y. He. Optimal Deployment of Dynamic Wireless Charging Facilities for an Electric Bus System. Transportation Research Record: Journal of the Transportation Research Board, 2017. 2647: 100-108.

3. Kunith, A., R. Mendelevitch, A. Kuschmierz, and D. Goehlich. Optimization of Fast Charging Infrastructure for Electric Bus Systems-Electrification of a City Bus Network. Proc., 29th International Electric Vehicle Symposium (EVS29), EDTA, Washington, D.C., 2016, pp. 1-12.

4. Eudy, L., R. Prohaska, K. Kelly, and M. Post. Foothill Transit Battery Electric Bus Demonstration Results. NREL/TP-5400-65274. National Renewable Energy Laboratory, Golden, CO, November 2015.

5. Miles, J., and S. Potter. Developing a Viable Electric Bus Service: The Milton Keynes Demonstration Project. Research in Transportation Economics, Vol. 48, 2014, pp. 357-363.

6. Li, J. Q. Battery-electric Transit Bus Developments and Operations: A Review. International Journal of Sustainable Transportation, Vol. 10, No. 3, 2016, pp. 157-169.

7. Kunith, A., R. Mendelevitch, and D. Goehlich. Electrification of a City Bus Network-An Optimization Model for Cost-effective Placing of Charging Infrastructure and Battery Sizing of Fast-charging Electric Bus Systems International Journal of Sustainable Transportation, Vol. 11, No. 10, 2017, pp. 707-720.

8. Ben-Tal, A., and A. Nemirovski. Robust Solutions of Uncertain Linear Programs. Operations Research Letters, Vol. 25, No. 1, 1999, pp. 1-13.
9. Ben-Tal, A., A. Goryashko, E. Guslitzer, and A. Nemirovski. Adjustable Robust Solutions of Uncertain Linear Programs. Mathematical Programming, Vol. 99, No. 2, 2004, pp. 351-376.

10. Ben-Tal, A., L. El Ghaoui, and A. Nemirovski. Robust Optimization. Princeton University Press, 2009.

11. Ben-Tal, A., B. Do Chung, S. R. Mandala, and T. Yao. Robust Optimization for Emergency Logistics Planning: Risk Mitigation in Humanitarian Relief Supply Chains. Transportation Research Part B: Methodological, Vol. 45, No. 8, 2011, pp. 1177-1189.

12. Lu, C. C. Robust Multi-period Fleet Allocation Models for Bike-sharing Systems. Networks and Spatial Economics, Vol. 16, No. 1, 2016, pp. 61-82.

13. Chung, D. B., T. Yao, C. Xie, and A. Thorsen. Robust Optimization Model for a Dynamic Network Design Problem Under Demand Uncertainty. Networks and Spatial Economics, Vol. 11, No. 2, 2011, pp. 371-389.

14. Liu, Z., and Z. Song. Robust Planning of Dynamic Wireless Charging Infrastructure for Battery Electric Buses. Transportation Research Part C: Emerging Technologies, Vol. 83, 2017, pp. 77-103.

15. Qin, N., A. Gusrialdi, R. P. Brooker, and T. Ali. Numerical Analysis of Electric Bus Fast-charging Strategies for Demand Charge Reduction. Transportation Research Part A: Policy and Practice, Vol. 94, 2016, pp. 386-396.

16. Glineur, F. Topics in Convex Optimization: Interior-point Methods, Conic Duality and approximations. U Mons, Belgium, 2001, Chapter 3, pp. 65-66.

17. Wang, Y., J. Jiang, and T. Mu. Context-aware and Energydriven Route Optimization for Fully Electric Vehicles via Crowd Sourcing. Intelligent Transportation Systems, Vol. 14, No. 3, 2013, pp. 1331-1345.

18. SIRI VehicleMonitoring Call. SIRI VehicleMonitoring Request. http://developer.rideuta.com/DeveloperResources/ VehicleByVehcileInstructions.aspx (accessed April 19, 2018)

19. National Research Council. Transitions to Alternative Vehicles and Fuels. National Academies Press, Washington, D.C., 2013.

20. Schroeder, A., and T. Traber. The Economics of Fastcharging Infrastructure for Electric Vehicles. Energy Policy, Vol. 43, 2012, pp. 136-144.

21. California Environmental Protection Agency. Advanced Clean Transit Battery Cost for Heavy-Duty Electric Vehicles (Discussion Draft). Air Resources Board, Califonia, 2016.

22. Rosenthal, R. E. GAMS-A User's Guide. GAMS Development Corporation, Washington, D.C., 2012.

23. CPLEX, I. I. V12. 1: User's Manual for CPLEX. International Business Machines Corporation, Vol. 46, No. 53, 2009, p. 157.

The Standing Committee on Bus Transit Systems ( AP050) peerreviewed this paper (18-03360).

The views expressed are those of the authors and do not reflect the official policy or position of the project's sponsors. 